\title{
Comparative Study Between Erythrocytes Blood Cells and Modulated Circular Aperture Using Speckle Photography
}

\author{
Abdallah Mohamed Hamed ${ }^{1, *}$, Tarek Abd-Elmotelb Al-Saeed ${ }^{2}$ \\ ${ }^{1}$ Physics Department, Faculty of Science, Ain Shams University, Cairo, Egypt \\ ${ }^{2}$ Biomedical Department, Faculty of Engineering, Helwan University, Cairo, Egypt
}

Email address:

amhamed73@hotmail.com (A. M. Hamed)

${ }^{*}$ Corresponding author

\section{To cite this article:}

Abdallah Mohamed Hamed, Tarek Abd-Elmotelb Al-Saeed. Comparative Study Between Erythrocytes Blood Cells and Modulated Circular Aperture Using Speckle Photography. American Journal of Optics and Photonics. Vol. 7, No. 3, 2019, pp. 57-62.

doi: 10.11648/j.ajop.20190703.12

Received: August 3, 2019; Accepted: August 22, 2019; Published: October 9, 2019

\begin{abstract}
A manipulation of four circles arranged along the Cartesian coordinates in uniform circular aperture is compared with selected four blood cells forming the object using the technique of speckle photography. The aperture model is considered have two- fold symmetry. A diffuser is placed in the same plane of the object or the model of four circles in order to get the modulated speckle pattern which is the Fourier spectrum of object information convoluted with the Fourier spectrum of the random diffuser. The modulated speckle pattern is smeared by the Point Spread Function computed from the diffraction corresponding to the whole circular aperture. The reconstruction process gave nearly similar speckle images little degraded by the noise originated from the diffuser. The Point Spread Function corresponding to the model of four circles is computed analytically and also using the FFT technique. A comparison is made with the Fourier spectrum of the blood cells and its corresponding autocorrelation function giving nearly similar results. We can acquire the erythrocytes blood cells in the imaging plane by operating the FFT upon the modulated speckle images. The reconstructed images of blood cells are affected by the noise originated from the diffuser. A Mat- Lab code is made in the construction of the aperture and all the images computed in the processing.
\end{abstract}

Keywords: Two - Fold Symmetrical Aperture, Model of Four Circles as Aperture, Point Spread Function, Speckle Imaging

\section{Introduction}

A lot of work made by many authors applied on medical images concerning the speckle images used only circular window. For example, the image contrast is computed for the speckle images corresponding to the blood cells using conventional window Briers and Webster in [1-5]. The contrast $\mathrm{K}$ values are between 0 and 1 . If there is no or little motion to blur all the speckles, Goodman [6] showed that assuming ideal conditions for producing a speckle pattern (i.e., perfectly diffusing surface, polarized light, and absence of noise), the standard deviation and mean intensity will be same, and the speckle contrast is then equal to unity, which is the maximum value for the speckle contrast. Such types of speckle pattern are called "fully developed speckle". In contrast, a speckle contrast of zero means that the scatters are moving fast enough to blur all the speckles. Retinal bloodflow visualization by means of laser speckle photography [1] and other work on capillary blood flow was investigated [4]. Francon and Goodman [6, 7] published enormous work on laser speckle and its application in different fields.

In the past decade $[8,9]$ suggested the linear, the quadratic, and the $\mathrm{B} / \mathrm{W}$ apertures of constant annular width in the study of the Confocal Laser Scanning Microscope. They computed the Point Spread Function (PSF) and the image of a point in case of conventional and confocal microscopes and obtained resolution improvement compared with circular aperture manipulations. In addition, an arrangement of four circles and two annuli forming two- fold symmetry and another arrangement of four - fold is suggested [10] symmetry in order to get compromised improvement of resolution and contrast. Recently, discrimination between modified apertures is given $[11,12]$ for elliptic, kidney, graded index 
shapes using speckle images and the PSF is computed in all cases. In addition, discrimination between the malignant and benign mammographic images is attained using speckle imaging and correlation techniques [13]. Image analysis of modified Hamming aperture and its application on confocal microscopy and holography is presented [14]. Later, Linearquadratic aperture is investigated [15]. Recently, image processing of Erythrocytes blood cells using the B/W concentric annuli of different widths of annuli is investigated $[16,17]$ in the Confocal Scanning Laser Microscope.

In this study, we investigate the speckle images for the microscopic blood cells using apertures of two- fold symmetry composed of four circles instead of clear circular window. The obtained speckle images are discussed and the contrast is computed for the different images.

\section{Theoretical Analysis}

A modeling of the image of the Erythrocytes blood cells is presented. It consists of four equal circles placed along the Cartesian coordinates two circles are at distances $\left( \pm 3 / 4 \rho_{\max }\right.$, 0 ) along the $\mathrm{x}$ - axis, and the other two circles are at distances $\left(0, \pm 3 / 4 \rho_{\max }\right)$ along the $y$ - axis. The described circular aperture of radius $\rho_{\max }$ provided with the four circles each has radius $\rho_{\max } / 4$ is shown in the figure 1 . A matrix of 1024 $\times 1024$ pixels represent the whole image, where $\rho_{\max }=512$ pixels represent the radius of the external contour. While each of the four circles has radius $\rho_{\max } / 4=128$ pixels which placed symmetrically at distances $\pm 3 / 4 \rho_{\max }= \pm 384$ pixels.

The aperture shown in the figure 1 , is mathematically represented as follows:

$$
P(\rho)=\sum_{i=1}^{4} P_{i}(\rho) ; i=1,2, \ldots, 4
$$

Where $P_{i}(\rho)$ is written as follows:

$$
\mathrm{I}(\mathrm{r})=|\mathrm{h}(\mathrm{r})|^{2}=\left[\frac{4 \mathrm{~J}_{1}\left(\mathrm{w}_{1}\right)}{\mathrm{w}_{1}}\right]^{2}\{\cos
$$

Consider coherent illumination obtained using laser beam after it is spatially filtered. A randomly distributed function in the form $\mathrm{d}(\mathrm{x}, \mathrm{y})$ is coherently illuminated by the described aperture giving the transmitted function represented as follows:

$$
T(x, y)=P(\rho) \cdot d(\rho) ; \text { where } \rho=(x, y)
$$

The speckle pattern formed in the Fourier plane is computed by operating the Fourier transform upon equation (8), where the direct multiplication of the two functions is transformed into convolution product of the Fourier

$$
S(u, v)=\frac{4 J_{1}\left(w_{1}\right)}{w_{1}}\left\{\cos \left(\frac{3 \pi \rho_{\max } r \cos \phi}{2 \lambda f}\right)+\cos \left(\frac{3 \pi \rho_{\max } r \sin \phi}{2 \lambda f}\right)\right\} \otimes \check{d}(r)
$$

When the transmittance of the image of the blood cells has distribution A ( $x, y)$, then the speckle image is further modulated by the Fourier spectrum of the blood cells simultaneously with the PSF of the aperture. In this case, the speckle is represented as follows:

$$
\begin{gathered}
\mathrm{P}_{\mathrm{i}}(\rho)=1 \text { for }\left|\rho-\rho_{\mathrm{i}}\right| \leq \frac{\rho_{\max }}{4} \\
\mathrm{P}_{\mathrm{i}}(\rho)=0 ; \text { Otherwise }
\end{gathered}
$$

The Fourier spectrum or the Point Spread Function (PSF) is calculated by operating the Fourier transform upon equation (1) considering circular symmetry of revolution to get the following result:

$$
\begin{gathered}
h(r)=\frac{2 J_{1}\left(w_{1}\right)}{w_{1}} \sum_{\mathrm{i}=1}^{4} \exp \left(-\mathrm{jk} \rho_{i} r / f\right) \text {; where } \mathrm{k}=2 \pi / \lambda \text { and } \\
\mathrm{w}_{1}=\frac{\mathrm{k} \rho_{\max } r}{4 f} \\
h(r)=\frac{2 J_{1}\left(w_{1}\right)}{w_{1}}\left\{\begin{array}{c}
\exp \left(-\frac{3 \mathrm{jk} x_{0} u}{4 f}\right)+\exp \left(+\frac{3 \mathrm{jk} x_{0} u}{4 f}\right)+ \\
\exp \left(-\frac{3 \mathrm{jk} y_{0} v}{4 f}\right)+\exp \left(+\frac{3 \mathrm{jk} y_{0} v}{4 f}\right)
\end{array}\right\} \text { (4) }
\end{gathered}
$$

Since the circles are arranged symmetrically along the coordinates and have equal radii, then

$x_{0}=y_{0}=\rho_{\max }$, where the radial coordinate in the Fourier plane is $r=(u, v)$.

Consequently, we obtain, using equation (4), the following result for the PSF:

$$
h(r)=\frac{4 J_{1}\left(w_{1}\right)}{w_{1}}\left\{\cos \left(\frac{3 \pi \rho_{\max } r \cos \phi}{2 \lambda f}\right)+\cos \left(\frac{3 \pi \rho_{\max } r \sin \phi}{2 \lambda f}\right)\right\}(5)
$$

where $\mathrm{u}=\mathrm{r} \cos \phi$, and $\mathrm{v}=\mathrm{r} \sin \phi$. With $\phi$ azimuthal coordinate in the Fourier plane $(r, \phi)$.

At $\phi=0$, the PSF is reduced to the following:

$$
h(r)=\frac{4 J_{1}\left(w_{1}\right)}{w_{1}}\left\{\cos \left(\frac{3 \pi \rho_{\max } r}{2 \lambda f}\right)\right\}=\frac{4 J_{1}\left(w_{1}\right)}{w_{1}}\left\{\cos \left(3 w_{1}\right)\right\}(6)
$$

The corresponding intensity for certain $\phi$ is calculated as follows:

$\left.\left(\frac{3 \pi \rho_{\max } r \cos \phi}{2 \lambda f}\right)+\cos \left(\frac{3 \pi \rho_{\max } r \sin \phi}{2 \lambda f}\right)\right\}^{2}$

transform corresponding to each function. Hence, we get:

$$
\begin{aligned}
S(u, v)= & \text { F. T. }\{T(x, y)\}=\text { F. T. }\{P(\rho) \cdot d(\rho)\}= \\
& \text { F. T. }\{P(\rho)\} \otimes \text { F.T. }\{d(\rho)\}
\end{aligned}
$$

The Fourier transform of the diffuser function give the ordinary speckle image which is convolved with the Fourier spectrum of the aperture or the PSF described by equation (5). Hence the complex amplitude of the formed speckle, which is smeared by the PSF corresponding to the aperture, is computed giving the following result:

$$
\begin{aligned}
S_{\text {blood }}(\mathrm{u}, \mathrm{v}) & =\text { F.T. }\left\{T_{\text {blood }}(\mathrm{x}, \mathrm{y})\right\}=\text { F. T. }\{\mathrm{A}(\rho) \cdot \mathrm{P}(\rho) \cdot \mathrm{d}(\rho)\} \\
& =\text { F. T. }\{\mathrm{A}(\rho)\} \otimes \text { F. T. }\{\mathrm{P}(\rho)\} \otimes \text { F.T. }\{\mathrm{d}(\rho)\}
\end{aligned}
$$

Substitute equation (10) in equation (11), we get the 
following result for the speckle in presence of the blood cells:

$$
\begin{gathered}
S_{\text {blood }}(\mathrm{u}, \mathrm{v})=\mathrm{F} . \mathrm{T} .\{\mathrm{A}(\rho)\} \otimes S(u, v)=\check{A}(r) \otimes S(r) ; \\
\text { where } \mathrm{r}=(\mathrm{u}, \mathrm{v}) .
\end{gathered}
$$

We reconstruct the image of the aperture and the image of the blood cells by operating the inverse Fourier transform upon equation (10) and equation (12) independently. The

$$
S_{\text {blood }}(\mathrm{u}, \mathrm{v})=\text { F. T. }\{\mathrm{A}(\rho)\} \otimes \check{d}(r)=\check{A}(r) \otimes \check{d}(r) ; \text { where } \mathrm{r}=(\mathrm{u}, \mathrm{v}) .
$$

The speckle contrast defined as the ratio of the standard deviation $\sigma$ to the mean intensity $<\mathrm{I}>$ Briers and Webster [3]:

$$
K=\frac{\sigma}{<I>}=\frac{\sqrt{\sum\left(I_{i, j}-<I>\right)^{2}}}{<I>}
$$

The standard deviation and mean of the pixel intensity in (14) are computed in a neighborhood $\mathrm{N}$ around the pixel $\mathrm{P}$ ( $\mathrm{x}$, $\mathrm{y})$ in the image matrix of dimensions $\mathrm{N} \times \mathrm{N}$.

In general, the contrast of an image is computed for a matrix of dimensions $\mathrm{N} \times \mathrm{M}$. from the root mean square value or the standard deviation as follows:

$$
\begin{aligned}
\text { contrast } & =\sqrt{ } \frac{1}{\mathrm{NMP}} \sum_{i=1}^{M} \sum_{j=1}^{N} \sum_{k=1}^{P}[I(i, j, k)-B]^{2} \\
\text { Where } B & =\sqrt{ } \frac{1}{\mathrm{NMP}} \sum_{i=1}^{M} \sum_{j=1}^{N} \sum_{k=1}^{P}[I(i, j, k)]
\end{aligned}
$$

\section{Results and Discussions}

We consider the shape of the model of four apertures located symmetrically in an image as in Figure 1. The diameters of each circular aperture are 256 pixels and the center of each circular apertures is 383 pixels from the center of the image. The dimension of the whole image is $1024 \times 1024$.

We apply a diffuser to the image and obtained the speckle pattern in Figure 2. A crowded grid structure can be seen in the image which is originated from interference of four circular apertures. The grid structure can be seen in the areas of the circular apertures. The image appears noisy with salt and pepper noise. The image of the speckle pattern is $256 \times 256$ pixels.

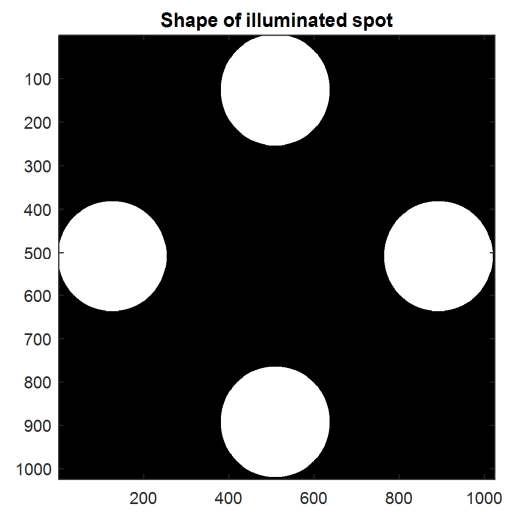

Figure 1. Shape of the model composed of four circles arranged symmetrically along the axes. Each circle has diameter $=256$ pixels and its center placed at a distance $=384$ pixels from the center of the whole image of dimensions $1024 \times 1024$ pixels. obtained images are affected by the noise originated from the diffuser.

In absence of the aperture, equation (12) gives speckle which is the convolution of the ordinary speckle corresponding to the diffuser and the Fourier spectrum corresponding to the blood cells. Hence, we get the following result:

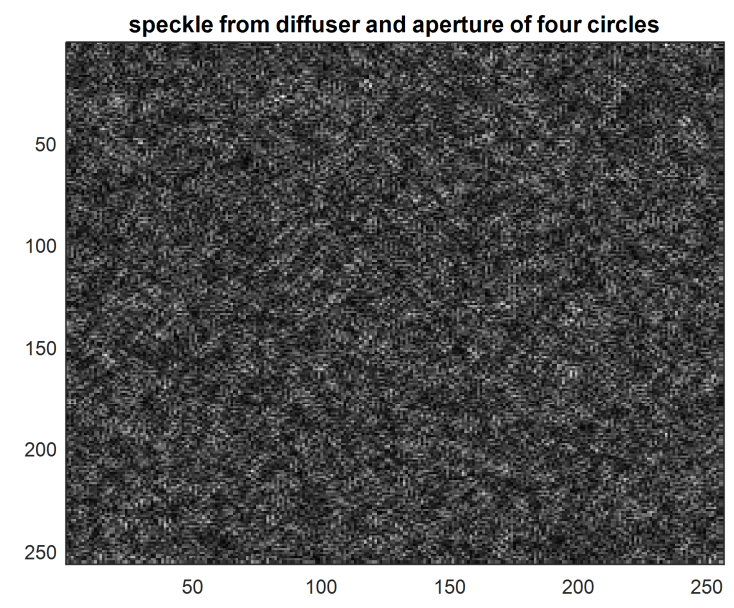

Figure 2. The speckle pattern obtained from the illuminated four circles superimposed over the diffuser. The speckle matrix has dimensions of $256 \times$ 256 pixels. A crowded grid structure is shown in the image which is originated from the interference of the four circles. The grid structure is shown crowded for greater areas of the circles.

In Figure 3, we draw the same image with four symmetric apertures and an image of erythrocytes having only four cells. The dimensions of erythrocytes blood cells are nearly analogous to that of the four circular apertures. Each circle has diameter of 256 pixels and the center of each is at 256 pixels from the center of the whole image. The dimension of the image is $1024 \times 1024$ pixels.
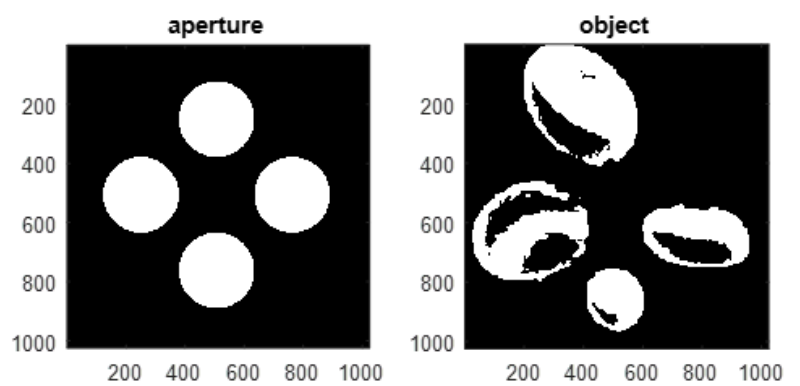

Figure 3. Shape of the model for aperture composed of four circles arranged symmetrically along the axes. Each circle has diameter $=256$ pixels and its center placed at a distance $=256$ pixels from the center of the whole image of dimensions $1024 \times 1024$ pixels. The object has four blood cells of the same dimensions like the aperture.

In Figure 4, we consider applying a diffuser to the aperture alone and applying diffuser to the erythrocytes image. Then we apply diffuser to both the aperture and the erythrocytes at the time and obtain the speckle pattern. The speckle pattern in the middle for the case of erythrocytes is noisy like salt 
and pepper noise. For the case of four circular apertures to the left the speckle pattern appears brighter and solid. To the right the speckle is more- brighter and more solid for the case of circular apertures and the erythrocytes. In addition, grid structure is shown in the speckle formed from the diffuser, aperture, and the 4 cells of the object which is attributed to the interference between the blood cells and the 4 circles of the aperture. while a visual equality between the speckle originated using either the aperture or the object as shown in the figures as expected since both of the object and the aperture each has four circles or cells. The contrast computed for each speckle image using the definition of the SD or the root mean square value represented by the formula (15), where the speckle image matrix is $256 \times 256$. The corresponding contrast values from the left of the figure 4 . are as follows: $\mathrm{K}=0.5058,0.5140$, and 0.4882 . Consequently, the speckle obtained in presence of the blood cells appears brighter than the other speckle images.
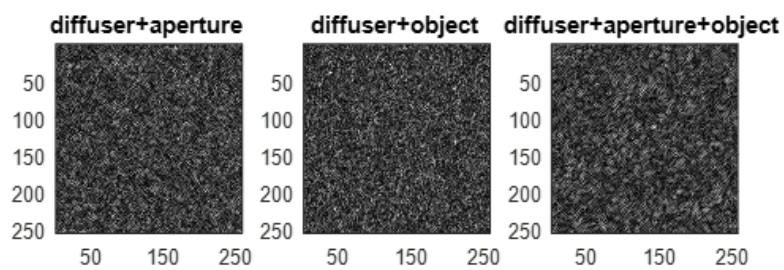

Figure 4. The speckle pattern corresponding to the diffuser superimposed over the aperture, diffuser superimposed over the object alone, and the last is a combination of both the object and aperture superimposed over the diffuser. The speckle images of dimensions $256 \times 256$ pixels.

In Figures (5-8), we consider line profile of the speckle pattern and get three profiles for different speckle images. In each figure of (5), (6), (7), and (8) we obtain speckle pattern corresponding to different images at $32,64,96$, and 128 pixels using the same diffuser. The figures show many peaks and troughs with different shapes giving discrimination between the speckle images. The maximum value is different in each case and the minimum value is always zero.
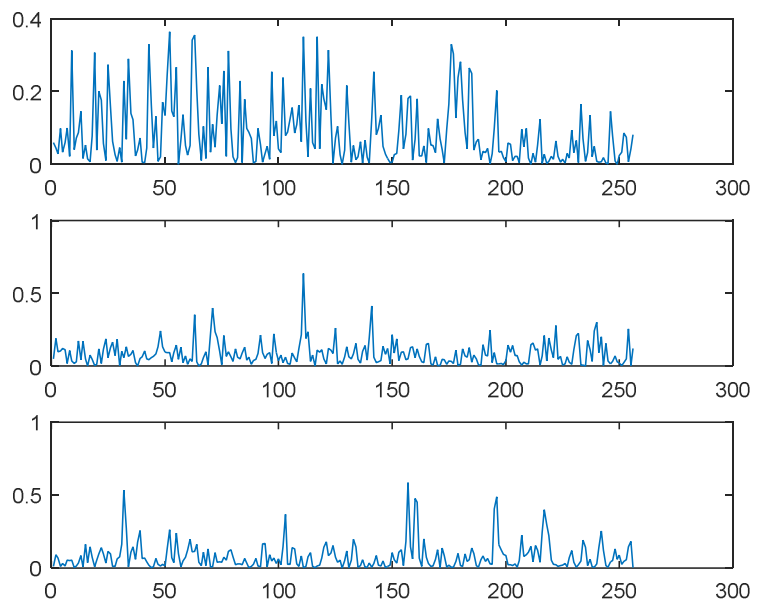

Figure 5. Line plot of the speckle images shown in the figure 4 at horizontal line at 32 pixels. Three different profiles are obtained for the different speckle images using the same randomly distributed function.
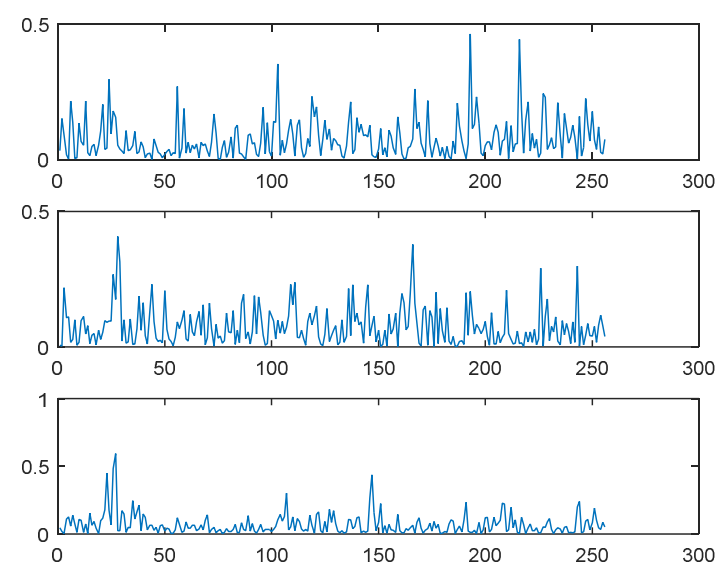

Figure 6. Line plot of the speckle images shown in the figure 4 at horizontal line at 64 pixels. Three different profiles are obtained for the different speckle images using the same randomly distributed function.
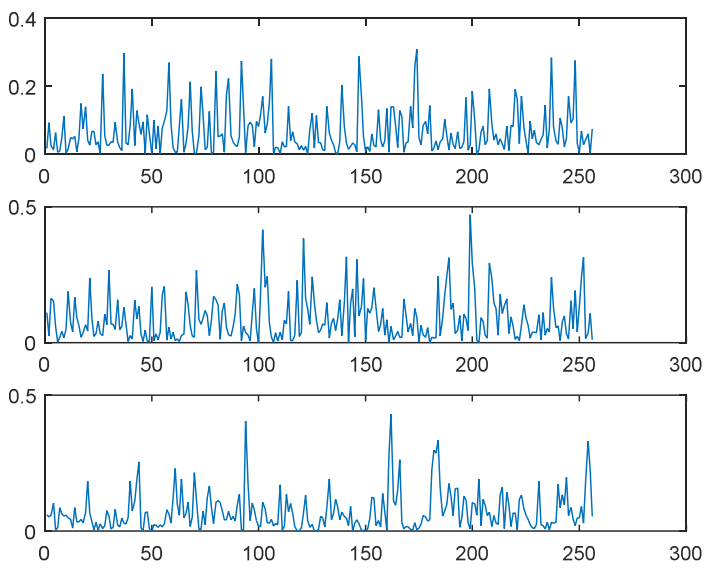

Figure 7. Line plot of the speckle images shown in the figure 4 at horizontal line at 96 pixels. Three different profiles are obtained for the different speckle images using the same randomly distributed function.
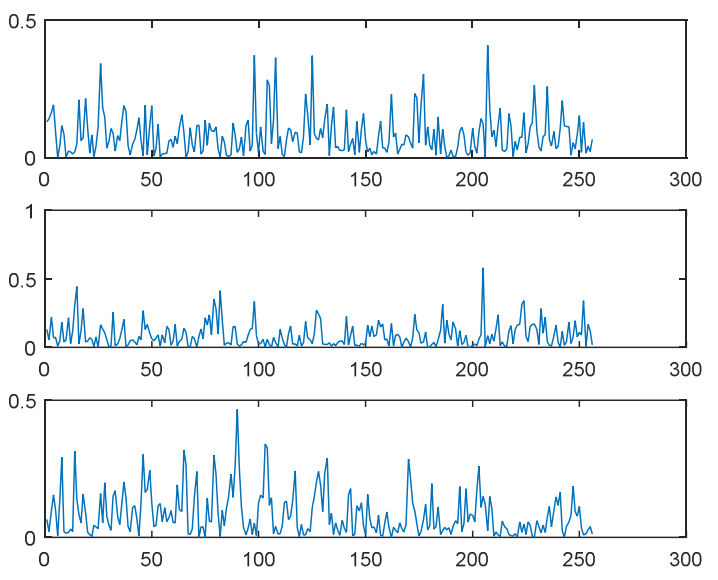

Figure 8. Line plot of the speckle images shown in the figure 4 at horizontal line at 128 pixels. Three different profiles are obtained for the different speckle images using the same randomly distributed function.

In Figure 9, we consider the image apertures and erythrocytes. To the complete image of both aperture and erythrocytes is reconstructed as in figure to the left. Circular apertures upon reconstruction appear distorted. In this image to the right we got segmentation of blood cells. The 
segmentation of the blood cells by the aperture is shown in the right figure since the blood cells are misaligned as compared with the four circles of the aperture. While complete images are shown in the left and the middle images corresponding to each of the aperture and the object.
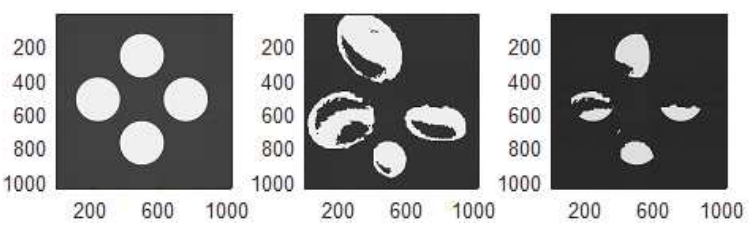

Figure 9. The reconstructed images corresponding to the speckle patterns shown in the figure 4.

In Figure (10), we consider the image of the FFT of the erythrocytes. The image of FFT shows peak at the center. Then we get some line profile of the spectrum which shows a peak. For figure (11) the peak is at 512 pixels and the value of the peak is -2.947 . For figure (12) the peak is at about 125 pixels its value is about -3 . The line profile shows a fluctuation about some certain curve.

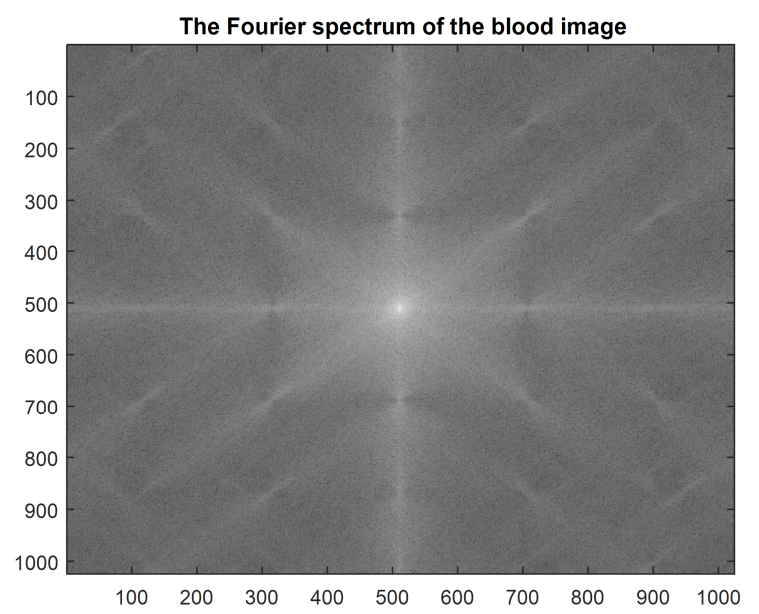

Figure 10. The image of the Fourier spectrum of the blood cells using FFT.

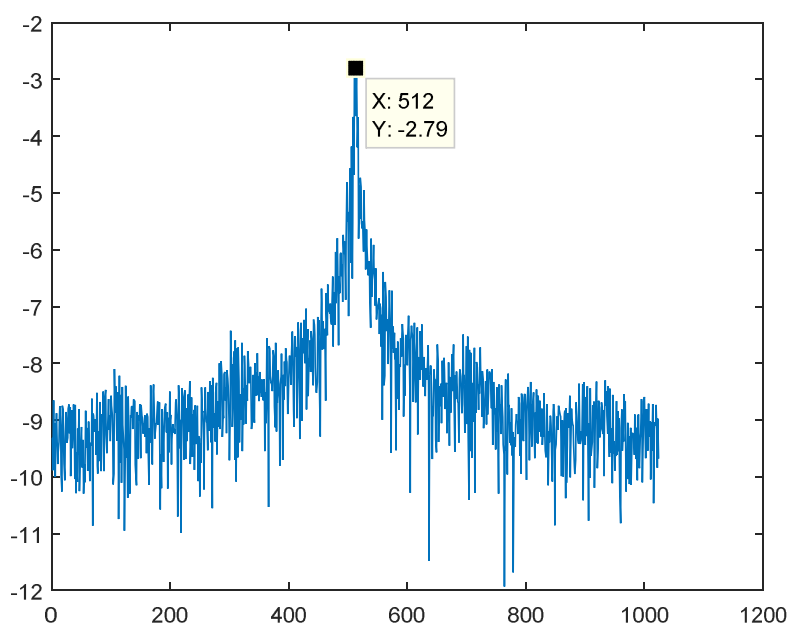

Figure 11. The profile of the Fourier spectrum corresponding to the image of the blood cells. The randomness in the profile distribution is due to the interference occurred from the neighboring blood cells. The profile is nearly similar to the model of the aperture composed of four circles.

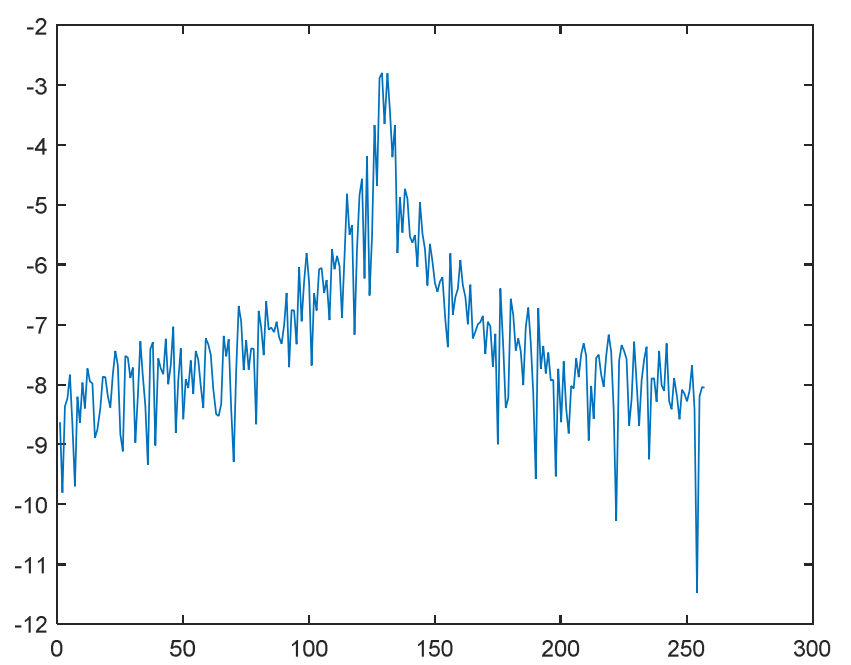

Figure 12. The profile of the Fourier spectrum, in the interval (384: 640) pixels, corresponding to the image of the blood cells. The randomness in the profile distribution is due to the interference occurred from the neighboring blood cells.

\section{Conclusion}

Firstly, it is shown that speckle images formed in case of either the aperture combined with the diffuser or the object and the diffuser or both of them are completely different.

Secondly, the speckle image in case of diffuser combined with the aperture and the object is brighter than that corresponding to the speckle image obtained in absence of the object. While the speckle formed from the diffuser and the object in absence of aperture showed a noisy pattern like salt- pepper noise. This information is justified from the image contrast results.

Thirdly, the line plot at certain horizontal line in the different speckle images showed different irregular shapes for all. The difference between the speckle images is originated from the convolution product of the ordinary speckle due to the diffuser and the Fourier spectrum of either the object or the aperture or both.

\section{References}

[1] Briers, J. D. and Fercher, A. F. (1982). Retinal blood-flow visualization by means of laser speckle photography. Invest. Ophthalmology Vis. Sci., 22 (2) 255-259.

[2] Briers, J. D. and Webster, S. (1995). Quasi real-time digital version of single-exposure speckle photography for full-field monitoring of velocity or flow fields. Opt. Communication, 116 (1) 36-42.

[3] Briers, J. D. and Webster, S. (1996). Laser speckle contrast analysis (LASCA): a non- scanning, full-field technique for monitoring capillary. J. Biomed. Opt., 1 (2) 174-179.

[4] Briers, J. D. Richards, G., and He. X. W. (1999). Capillary blood flow monitoring using laser speckle contrast analysis (LASCA). J. Biomed. Optic., 4 (1) 164-175.

[5] Briers, J. D. (2007). Laser speckle contrast imaging for measuring blood flow. Optica Applicata, 37 (1/2): 139-152. 
[6] Goodman, J. W. (1975). Statistical properties of laser speckle patterns. In Laser Speckle and Related Phenomena, volume 9, chapter 2, pages 9-75. Springer-Verlage.

[7] Francon, M. (1979). Laser Speckle and Applications in Optics. ISBN:9780323160728. Publisher: Academic Pr.

[8] Hamed, A. M. \& Clair, J. J. (1983). Image and superresolution in optical coherent microscopes. Optik 64 (4) 277284.

[9] Hamed, A. M. \& Clair, J. J. (1983). Studies on optical properties of confocal scanning optical microscope using pupils with radially transmission $\rho^{\mathrm{n}}$ distribution. Optik 65 (3) 209-218.

[10] Hamed, A. M. (1984). Resolution and Contrast in Confocal Optical Scanning Microscope, Optics \& Laser Tech. 16 (4) 93-96.

[11] Hamed, A. M. (2011). Discrimination between speckle images using diffusers modulated by some deformed apertures: Simulations. Journal of Optical Engineering 50 (1) 1-7. http://dx.doi.org/10.1117/1.3530085.
[12] Hamed, A. M. (2014). Study of graded index and truncated apertures using speckle images. Precision Instrument and Mech. PIM 3 (1) 144-152.

[13] Hamed, A. M. and Al-Saeed, T. A. (2014). Processing of mammographic images using speckle technique. International Journal of Computational Engineering IJCER, 4 (1) 56-62.

[14] Hamed, A. M. \& Al-Saeed, T. A. (2015). Image analysis of modified Hamming aperture: Application on confocal microscopy and holography. Journal of Modern Optics 62 (10) 801-810. http://dx.doi.org/10.1080/09500340.2015.1007102.

[15] Hamed, A. M. (2017). Improvement of point spread function (PSF) using linear- quadratic aperture. Optik, 131 (2) 838-849.

[16] Hamed, A. M. (2015). Topics on Optical and Digital Image Processing Using Holography and Speckle Techniques. I. S. B. N. 9781329328464- $1^{\text {st }}$ edition. Publisher: www.lulu.com.

[17] Hamed, A. M. (2017). The PSF of some modulated apertures (Application on speckle and interferometry images). ISBN: 9786202070706. Publisher: www.lap.com/. 